\title{
HD 8801: A UNIQUE SINGLE Am STAR WITH $\gamma$ DORADUS AND $\delta$ SCUTI PULSATIONS
}

\author{
Gregory W. Henry ${ }^{1}$ and Francis C. Fekel ${ }^{1,2}$ \\ Center of Excellence in Information Systems, Tennessee State University, 330 10th Avenue North, Nashville, TN 37203; \\ henry@schwab.tsuniv.edu, fekel@evans.tsuniv.edu \\ Received 2004 October 8; accepted 2004 December 20
}

\begin{abstract}
We have discovered the first example of a star pulsating intrinsically with both $\gamma$ Doradus and $\delta$ Scuti frequencies. The star, HD 8801, is an Am metallic-line star that appears to be single. Since the vast majority of Am stars are members of binary systems with periods less than 1000 days and also do not pulsate, HD 8801 possesses a very unusual, if not unique, combination of physical properties. Our photometry, acquired with an automatic telescope at Fairborn Observatory, resolves six independent pulsation periods between 0.048 and 0.404 days. The two longest periods lie within the range of $\gamma$ Dor variables, while the four shorter periods are within the range of $\delta$ Sct stars. Eleven radial velocities, obtained over an 11 month period, have a mean of $0.8 \pm 0.3 \mathrm{~km} \mathrm{~s}^{-1}$, providing no evidence for a spectroscopic companion. The star is on the main sequence and lies within both the $\gamma$ Dor and $\delta$ Sct instability strips. Pulsation constants computed for the six periods agree with pulsation constants computed for other $\gamma$ Dor and $\delta$ Sct variables. HD 8801 is also the first confirmed case of a metallic-line star pulsating with $\gamma$ Dor frequencies. Its projected rotational velocity of $55 \mathrm{~km} \mathrm{~s}^{-1}$ is typical for Am stars, which, as a group, rotate more slowly than normal A stars. HD 8801's slow rotation compared to normal A stars does not appear to arise from either a binary companion or evolutionary expansion and thus remains a puzzle. The coexistence of metallicism and pulsation in several main-sequence stars near the red edge of the $\delta$ Sct strip contradicts recent models that predict pulsations only in more evolved stars.
\end{abstract}

Key words: $\delta$ Scuti — stars: chemically peculiar — stars: early-type — stars: fundamental parameters stars: oscillations - stars: variables: other

Online material: machine-readable table

\section{INTRODUCTION}

The $\gamma$ Doradus variables have been recognized only recently as a new class of pulsating stars with photometric and spectroscopic variations arising from nonradial, gravity $(g)$ mode pulsations of high order $(n)$ and low spherical degree $(l)$ (Kaye et al. 1999). The most recent list of confirmed $\gamma$ Dor stars, given by Henry \& Fekel (2003), contains 42 entries. These stars typically exhibit multiple photometric periods between 0.3 and 3 days (Kaye et al. 1999) and sinusoidal light curves with amplitudes of a few millimagnitudes to a few percent (Henry \& Fekel 2003). They lie in a fairly tight region of the H-R diagram, on or just above the main sequence, that partially overlaps the cool edge of the $\delta$ Scuti instability strip (Handler 1999; Henry \& Fekel 2003). A driving mechanism for the $\gamma$ Dor stars, first proposed by Guzik et al. (2000) and based on modulation of the radiative flux from the interior of the star by a relatively deep envelope convection zone, has been found by Warner et al. (2003) to be consistent with the observed location of the $\gamma$ Dor variables in the H-R diagram. In contrast to the $\gamma$ Dor stars, the $\delta$ Sct variables have shorter periods, generally in the range $0.02-0.25$ days, and photometric amplitudes of a few millimagnitudes to several tenths of a magnitude (Rodríguez et al. 2000). The majority of $\delta$ Sct stars are nonradial, low-order, low-degree, pressure $(p)$ mode pulsators (Breger 2000) and are driven by the $\kappa$-mechanism (Chevalier 1971).

\footnotetext{
${ }^{1}$ Senior Research Associate, Department of Physics and Astronomy, Vanderbilt University, Nashville, TN 37235.

2 Visiting Astronomer, Kitt Peak National Observatory, National Optical Astronomy Observatory, operated by the Association of Universities for Research in Astronomy, Inc., under cooperative agreement with the National Science Foundation.
}

Given the overlap between the $\gamma$ Dor and $\delta$ Sct stars in the $\mathrm{H}-\mathrm{R}$ diagram, interesting questions arise concerning the relationship between these two classes of variable stars. In particular, can self-excited $\gamma$ Dor and $\delta$ Sct pulsations exist simultaneously in the same star? Breger \& Beichbuchner (1996) examined this question by searching the literature on $300 \delta$ Sct stars for evidence of additional low-frequency variations. Fewer than $10 \%$ of the stars in their study had reported evidence of additional low frequencies, and most of those could be dismissed as being due to variable comparison stars or instrumental/atmospheric effects. They were unable to establish definitively any example of the existence of both high and low pulsation frequencies in the same star.

Handler \& Shobbrook (2002) conducted a photometric search for stars with both $\gamma$ Dor and $\delta$ Sct pulsations, pointing out the exciting implications this would have for astroseismology and for the theoretical understanding of the $\gamma$ Dor driving mechanism if such stars could be found. They monitored a sample of 26 candidate $\gamma$ Dor stars, several of which they confirmed as true $\gamma$ Dor variables located within the $\delta$ Sct instability strip. Only one star, HD 209295, exhibited both types of pulsation, but the $\gamma$ Dor pulsations in this eccentric close binary are most likely excited by tidal effects (Handler et al. 2002). An additional star, HD 207651, was found to have clear $\delta$ Sct pulsations, as well as lower frequency variations, but they suspected the lower frequencies might be due to ellipsoidal effects in a close binary. The lack of any self-excited $\gamma$ Dor variables lying within the $\delta$ Sct strip that also possess $\delta$ Sct pulsations led Handler \& Shobbrook (2002) to conclude that $\gamma$ Dor stars are less likely to be $\delta$ Sct pulsators than are non- $\gamma$ Dor stars in the same temperature range. They pointed out that there is very little overlap in the period ranges of the two pulsation types and that there is 
$\gamma$ DOR AND $\delta$ SCT PULSATIONS IN HD 8801

TABLE 1

Photometric ObSeRVATIONS OF HD 8801

\begin{tabular}{|c|c|c|c|c|}
\hline Date (HJD - 2,400,000) & $\begin{array}{c}(P-C 1)_{b} \\
(\mathrm{mag})\end{array}$ & $\begin{array}{c}(P-C 1)_{y} \\
(\mathrm{mag})\end{array}$ & $\begin{array}{c}(C 1-C 2)_{b} \\
(\mathrm{mag})\end{array}$ & $\begin{array}{c}(C 1-C 2)_{y} \\
(\mathrm{mag})\end{array}$ \\
\hline $52961.5631 \ldots \ldots \ldots$ & -0.7739 & -0.8123 & 0.2268 & 0.2830 \\
\hline $52961.5742 \ldots \ldots \ldots$ & -0.7826 & -0.8175 & 0.2308 & 0.2821 \\
\hline $52961.5796 \ldots \ldots \ldots \ldots \ldots \ldots \ldots \ldots . .$. & -0.7777 & -0.8150 & 0.2311 & 0.2844 \\
\hline $52961.5849 \ldots \ldots \ldots \ldots \ldots \ldots \ldots \ldots \ldots$ & -0.7770 & -0.8150 & 0.2330 & 0.2861 \\
\hline 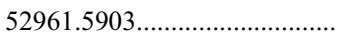 & -0.7720 & -0.8082 & 0.2279 & 0.2799 \\
\hline $52961.5963 \ldots \ldots \ldots \ldots \ldots \ldots \ldots$ & -0.7738 & -0.8099 & 0.2286 & 0.2843 \\
\hline
\end{tabular}

Note.-Table 1 is published in its entirety in the electronic edition of the Astronomical Journal. A portion is shown here for guidance regarding its form and content.

a clear distinction between the pulsation constants of the two groups.

Henry et al. (2004) reported new photometric and spectroscopic observations of HD 207651 and confirmed the suggestion of Handler \& Shobbrook (2002) that the low frequencies in this $\delta$ Sct star result from the ellipticity effect, not from $\gamma$ Dor pulsations. They also used their larger sample of confirmed $\gamma$ Dor stars to strengthen the argument that $\gamma$ Dor and $\delta$ Sct pulsations do not coexist in the same star. Out of 21 carefully monitored $\gamma$ Dor stars also located within the $\delta$ Sct instability strip, none showed evidence for $\delta$ Sct pulsations. The incidence of $\delta$ Sct pulsations within the instability strip (Fig. 8 of Handler \& Shobbrook 2002) predicts that at least two or three of the $21 \gamma$ Dor stars should exhibit $\delta$ Sct variability if the two types of pulsation can exist independently. To date, no examples of selfexcited $\gamma$ Dor and $\delta$ Sct pulsations in the same star have been established (e.g., Kurtz 2002, 2003).

This work continues a series of papers in which we have examined the spectroscopic and photometric characteristics of candidate $\gamma$ Dor stars (e.g., Henry \& Fekel 2003; Henry et al. 2004). Most of the stars we have confirmed as $\gamma$ Dor variables in this series have come from the list of prime candidates in Handler (1999). However, the subject of this paper, HD $8801=$ HR $418\left(\alpha=1^{\mathrm{h}} 27^{\mathrm{m}} 26^{\mathrm{s}} 7, \delta=+41^{\circ} 06^{\prime} 04^{\prime \prime}[\mathrm{J} 2000.0], V=6.42\right.$, $B-V=0.31$ ), comes from our own volume-limited photometric survey of $114 \gamma$ Dor candidates selected from the Hipparcos catalog (Perryman et al. 1997) to lie within the $\gamma$ Dor instability strip. It is one of 39 stars out of the 114 in the survey (G. W. Henry et al. 2005, in preparation) found to exhibit low-amplitude photometric variability and was also flagged as an "unresolved variable" in the Hipparcos catalog (Perryman et al. 1997). As described in the following sections, we have found it to be the first case of a star pulsating intrinsically with both $\gamma$ Dor and $\delta$ Sct frequencies. HD 8801 is also an Am metallic-line star that appears to be single, apart from a faint, distant visual companion, giving it a unique combination of physical properties.

\section{PHOTOMETRY}

\subsection{Observations}

The photometric observations in this paper were acquired on nine nights between 2003 November 17 and December 6 with the T12 $0.8 \mathrm{~m}$ automatic photoelectric telescope (APT) at Fairborn Observatory. The T12 APT is equipped with a twochannel precision photometer employing two EMI 9124QB bi-alkali photomultiplier tubes to make simultaneous measurements in the Strömgren $b$ and $y$ passbands. The telescope, photometer, observing procedures, and data reduction techniques employed with this APT were very similar to those for our T8 $0.8 \mathrm{~m}$ APT described in Henry (1999). The measurements of our program star HD $8801(P, V=6.42, B-V=0.31, \mathrm{~F} 1 \mathrm{~m})$ were made differentially with respect to the two comparison stars HD $8672(C 1, V=7.23, B-V=0.26$, F0) and HD 8007 $(C 2, V=6.95, B-V=0.35, \mathrm{~F} 0)$. HD 8801 is the primary of a visual double system (ADS $1151 \mathrm{AB}$ ); the secondary is $7 \mathrm{mag}$ fainter and $15^{\prime \prime}$ distant from HD 8801 and was included in the $45^{\prime \prime}$ diaphragm used to make the observations. However, the resulting dilution in the light of HD 8801 is insignificant. The observations were corrected for differential extinction with nightly extinction coefficients and transformed to the Strömgren system with yearly mean transformation coefficients. The external precision of our differential magnitudes, as determined from observations of pairs of constant stars, is $\sim 0.0015 \mathrm{mag}$ for this telescope. Further information on the automated telescopes can be found in Eaton et al. (2003).

The 473 individual $P-C 1$ and $C 1-C 2$ differential magnitudes in the Strömgren $b$ and $y$ passbands are listed in Table 1. The $b$ observations from all nine nights are plotted in Figure 1 (top). The nine nights are plotted individually in the other four panels of Figure 1 and in the five panels of Figure 2. The nightly light curves clearly show short-term variability superposed on longer term brightness changes.

\subsection{Period Analysis}

We applied the method of Vanicek (1971), as described in Henry et al. (2001), to search for periodicities in the photometric data. We first analyzed the $C 1-C 2$ comparison star data over the frequency range $0.05-30.0 \mathrm{day}^{-1}$, which corresponds to the period range $0.033-20$ days, and found no evidence for periodicity. The standard deviations of the $C 1-C 2$ observations are 0.0018 and $0.0020 \mathrm{mag}$ in the $b$ and $y$ bands, respectively, so there is no evidence of variability in either comparison star. However, the standard deviations of the $P-C 1$ differential magnitudes in $b$ and $y$ are 0.0062 and 0.0050 , respectively, clearly indicating low-amplitude variability in the program star. We analyzed the $P-C 1$ differential magnitudes over the same frequency range as the comparison stars. Because of the 1 week gap between our sixth and seventh nights, aliasing made it difficult to identify the correct frequencies. Therefore, we restricted our analysis to the first six nights, acquired within a seven-night interval. The resulting power spectra for the $b$ observations are shown in Figure 3, and the photometric results from the analysis of this six-night subset of our data are summarized in Table 2.

Figure 3 (top) shows three clusters of frequencies centered near 3,8 , and 20 day $^{-1}$, suggesting the possibility that HD 8801 displays both $\gamma$ Dor and $\delta$ Sct variability. The first six panels show the results of successively fixing the strongest detected frequencies in each panel. No further frequencies can be clearly identified in the bottom panel. The rms of the residuals after 

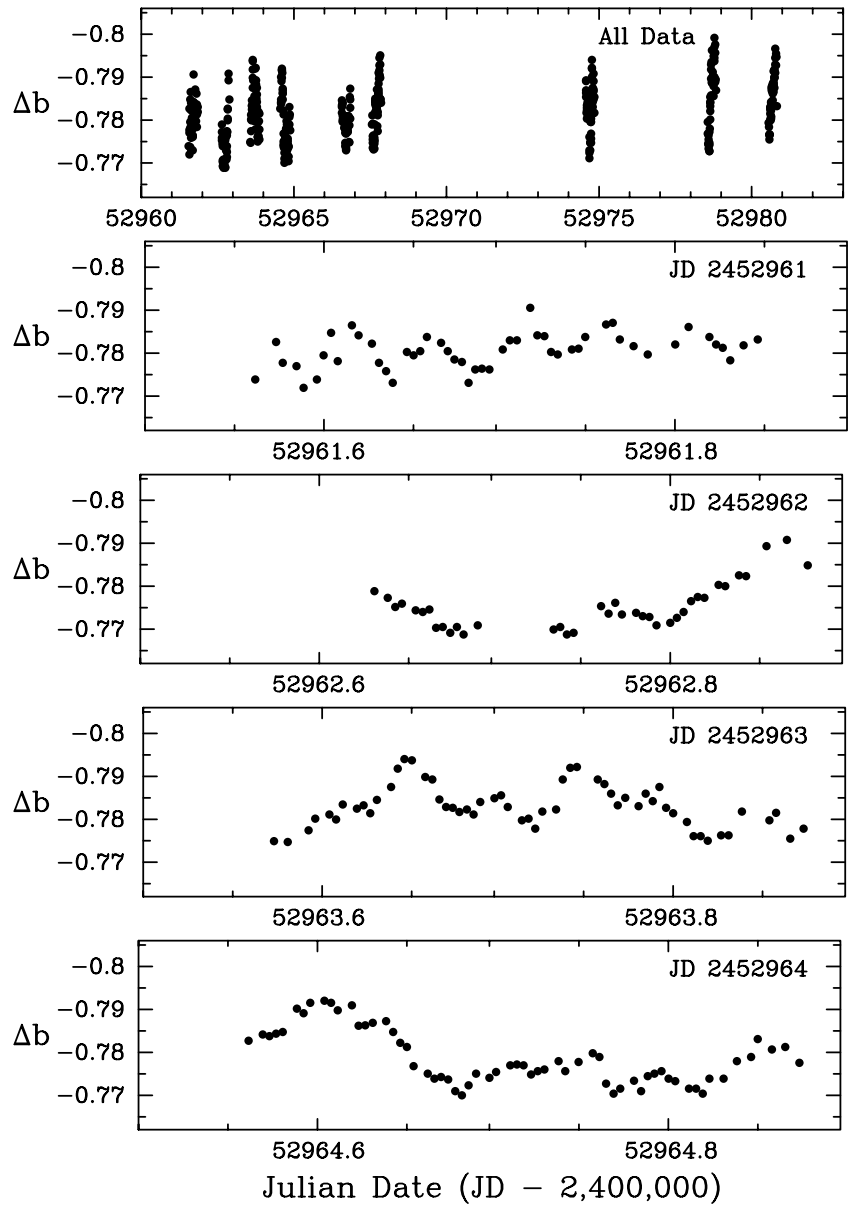

FIG. 1.-Complete set of Strömgren $\Delta b$ photometric observations of HD 8801 acquired on nine nights during 2003 November and December (top). The observations from the first four individual nights are replotted separately in the other four panels.

fixing the six frequencies identified in the first six panels is only $0.0024 \mathrm{mag}$ in $b$, indicating very little remaining variability beyond that accounted for by the six frequencies. As the results in Table 2 show, each of the three frequency clusters has been resolved into two closely spaced components; the lowest frequency pair is characteristic of $\gamma$ Dor pulsations, while the two higher frequency pairs are in the range of $\delta$ Sct pulsations. The criterion of Loumos \& Deeming (1978) gives a minimum frequency resolution of 0.24 day $^{-1}$ for our six-night data set; the three pairs of frequencies in Table 2 have separations of 0.36 , 0.53 , and $0.52 \mathrm{day}^{-1}$, so they are fully resolved in our analysis.

As shown in Table 2, we obtained essentially identical results in our analysis of the Strömgren $y$ data. The peak-to-peak amplitudes reported in column (6) are determined for each frequency without prewhitening for the other frequencies. The $b$ amplitudes average 1.32 times larger than the $y$ amplitudes (col. [7]), consistent with a pulsational origin for all frequencies. This is confirmed by the lack of short-period radial velocity variations (see $\S 3.2$ ), which rule out ellipsoidal effects in a binary system. Times of minimum light for each frequency are given in column (8); the times of minimum in the two passbands agree within their uncertainties, so there are no detectable phase shifts in our two-color photometry.

The six nights of $b$ data are phased with each of the six frequencies and times of minimum from Table 2 and plotted in Figure 4. In each panel, the data have been prewhitened to remove the other five frequencies to improve the visibility of the
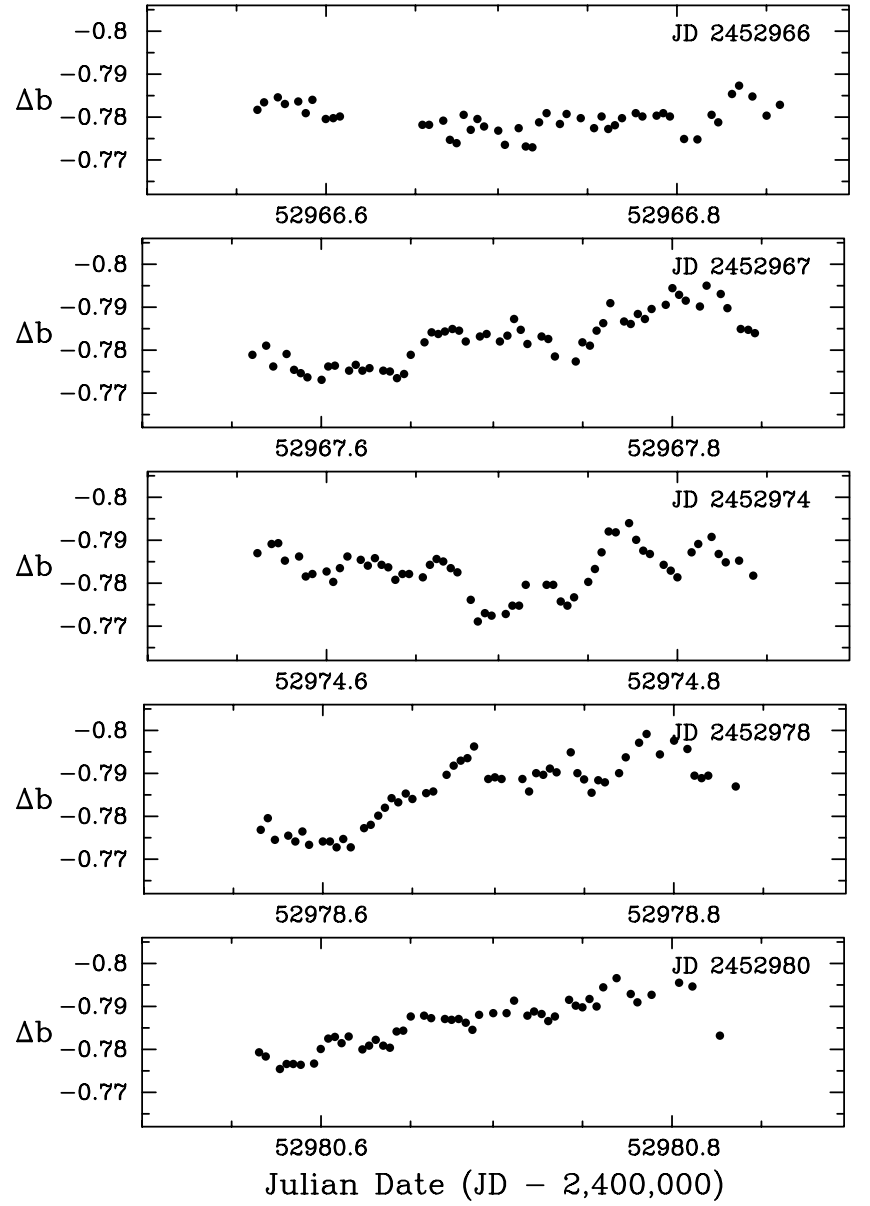

Fig. 2.-Strömgren $\Delta b$ photometric observations of HD 8801 from nights $5-9$ in the top panel of Fig. 1, replotted here in separate panels. The light curves clearly show short-term variability superposed on longer term brightness changes.

brightness variations. The light curves at all six frequencies are approximately sinusoidal.

\section{SPECTROSCOPY}

\subsection{Observations and Reductions}

Between 2003 October and 2004 September, seven spectroscopic observations were obtained at Kitt Peak National Observatory (KPNO) with the coudé feed telescope, the coudé spectrograph, and a TI CCD detector. The spectrograms are centered at $6430 \AA$, cover a wavelength range of about $80 \AA$, and have a 2 pixel resolution of $0.21 \AA$. The typical signal-tonoise ratio of our spectra is about 200 .

At our request, H. Abt (2003, private communication) acquired two additional spectra on consecutive nights in 2003 December with the same KPNO telescope and spectrograph but with the F3KB CCD detector. Those observations are centered at about $5360 \AA$ and have a resolution of $0.37 \AA$. In addition, G. Handler (2004, private communication) obtained two spectra of HD 8801 on a single night in 2004 July with the $2.7 \mathrm{~m}$ McDonald Observatory telescope, the echelle mode of the coudé spectrograph, and a $2048 \times 2048$ Tektronix CCD. The resolving power was 60,000 .

Our seven radial velocities (Table 3 ) were determined in the 6385-6444 A region with the IRAF ${ }^{3}$ cross-correlation program

\footnotetext{
${ }^{3}$ IRAF is distributed by the National Optical Astronomy Observatory.
} 


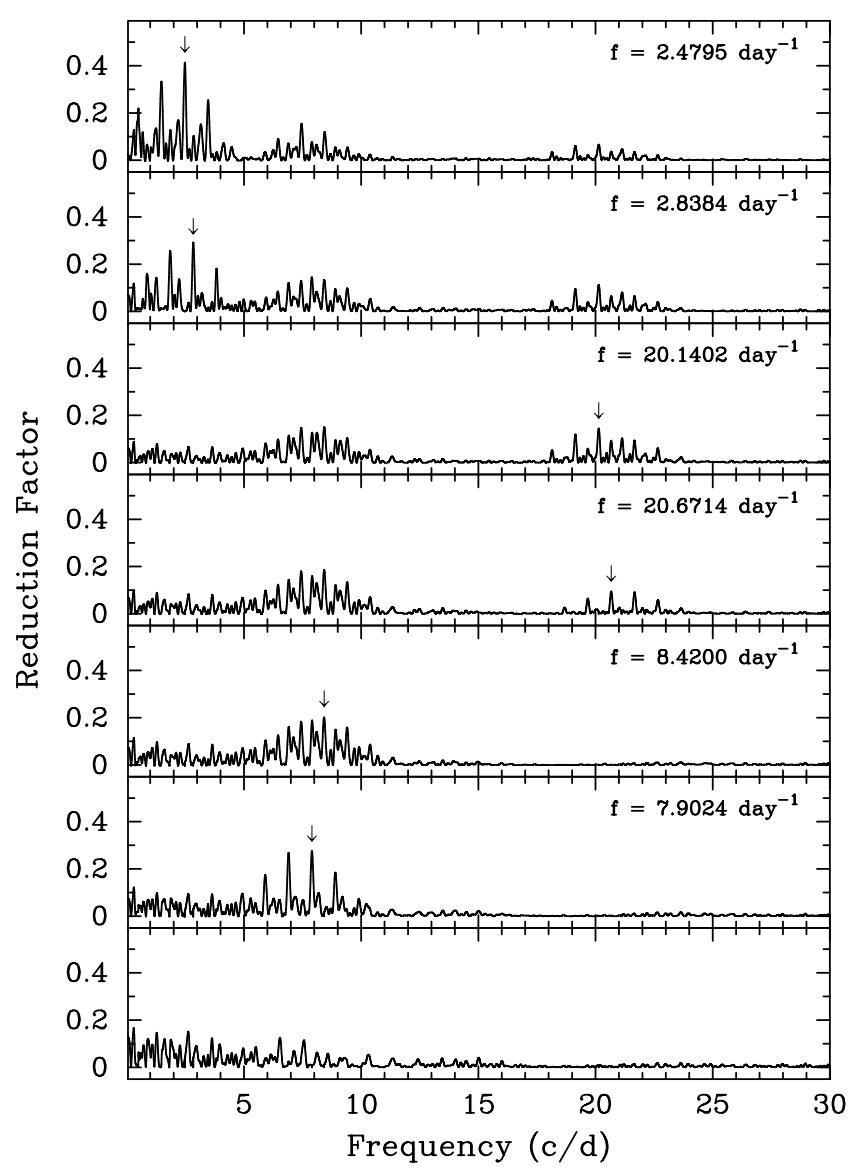

FIG. 3.-Power spectra of the first six nights of HD $8801 \Delta b$ photometry. Each frequency detected is marked with an arrow and then fixed for the analyses in subsequent panels. Values of the frequencies are given in the upper right corner of each panel (see Table 2). The first two (lowest) frequencies are within the range of typical $\gamma$ Dor pulsations; the last four (highest) frequencies are typical of $\delta$ Sct pulsations.

FXCOR (Fitzpatrick 1993). The IAU radial velocity standards HR 7560, $\iota$ Psc, and 10 Tau were used as reference stars. Their velocities of $0.0,5.6$, and $27.9 \mathrm{~km} \mathrm{~s}^{-1}$, respectively, were adopted from Scarfe et al. (1990). Velocities of Abt's spectra were measured by D. Willmarth (2003, private communication). Handler's two echelle spectra contain numerous orders, but only those cov-

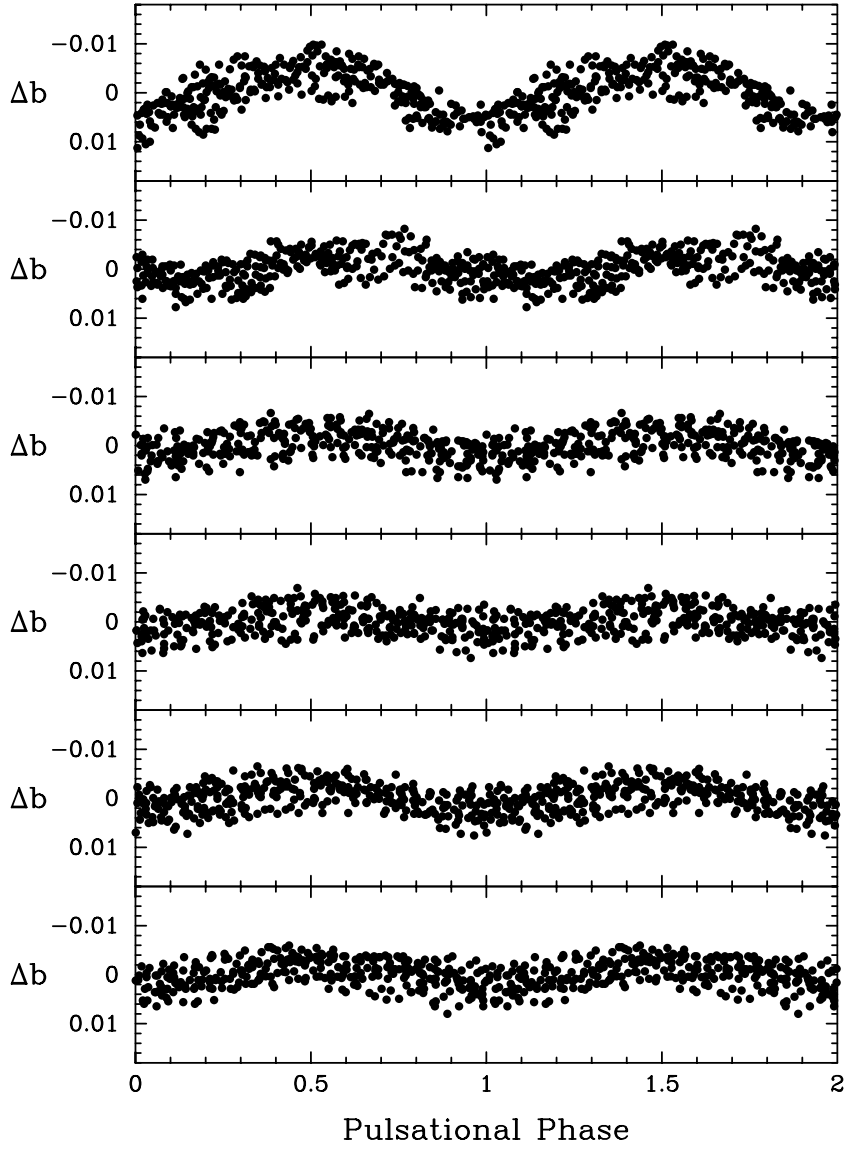

FIG. 4.-First six nights of HD $8801 \Delta b$ photometry, phased with the six frequencies and times of minimum from Table 2. Frequencies are 2.4795, $2.8384,20.1402,20.6714,8.4200$, and 7.9024 day $^{-1}$ (top to bottom). For each panel, the data set has been prewhitened to remove the other five frequencies.

ering the wavelength region from 5800 to $6400 \AA$ were measured by J. Tomkin (2004, private communication). The four additional velocities are listed in Table 3.

\subsection{Velocity Variations}

The Am spectral classification of HD 8801 ( $(3.3)$ suggests that this star might be a relatively close binary. However, the 11 recent radial velocities ( Table 3 ) taken over a range of 337 days

TABLE 2

Photometric Results For HD 8801

\begin{tabular}{|c|c|c|c|c|c|c|c|}
\hline $\begin{array}{l}\text { Photometric } \\
\text { Band } \\
\text { (1) }\end{array}$ & $\begin{array}{c}\text { Date Range } \\
(\mathrm{HJD}-2,400,000) \\
(2)\end{array}$ & $\begin{array}{c}N_{\text {obs }} \\
\text { (3) }\end{array}$ & $\begin{array}{l}\text { Frequency } \\
\left(\text { day }^{-1}\right) \\
(4)\end{array}$ & $\begin{array}{l}\text { Period } \\
\text { (days) } \\
(5)\end{array}$ & $\begin{array}{l}\text { Peak-to-Peak } \\
\text { Amplitude } \\
\text { (mmag) } \\
\text { (6) }\end{array}$ & $\begin{array}{c}b / y \\
\text { Amplitude } \\
\text { Ratio } \\
\text { (7) }\end{array}$ & $\begin{array}{c}T_{\min } \\
(\mathrm{HJD}-2,400,000) \\
(8)\end{array}$ \\
\hline \multirow[t]{6}{*}{$b$} & $52,961.5631-52,967.8488$ & 314 & $2.4795 \pm 0.0044$ & $0.40331 \pm 0.00072$ & $10.7 \pm 0.7$ & $1.23 \pm 0.13$ & $52,965.150 \pm 0.004$ \\
\hline & & & $2.8384 \pm 0.0039$ & $0.35231 \pm 0.00048$ & $4.9 \pm 0.8$ & $1.48 \pm 0.38$ & $52,965.158 \pm 0.010$ \\
\hline & & & $20.1402 \pm 0.0043$ & $0.04965 \pm 0.00001$ & $4.1 \pm 0.9$ & $1.28 \pm 0.44$ & $52,965.017 \pm 0.002$ \\
\hline & & & $20.6714 \pm 0.0042$ & $0.04838 \pm 0.00001$ & $3.1 \pm 0.9$ & $1.35 \pm 0.59$ & $52,965.025 \pm 0.002$ \\
\hline & & & $8.4200 \pm 0.0048$ & $0.11876 \pm 0.00007$ & $5.4 \pm 0.9$ & $1.23 \pm 0.33$ & $52,965.023 \pm 0.003$ \\
\hline & & & $7.9024 \pm 0.0047$ & $0.12654 \pm 0.00008$ & $4.5 \pm 0.9$ & $1.36 \pm 0.41$ & $52,965.078 \pm 0.004$ \\
\hline \multirow[t]{6}{*}{$y \ldots \ldots \ldots \ldots \ldots \ldots \ldots$} & $52,961.5631-52,967.8488$ & 314 & $2.4782 \pm 0.0045$ & $0.40352 \pm 0.00073$ & $8.7 \pm 0.6$ & & $52,965.148 \pm 0.004$ \\
\hline & & & $2.8367 \pm 0.0039$ & $0.35252 \pm 0.00048$ & $3.3 \pm 0.7$ & & $52,965.157 \pm 0.012$ \\
\hline & & & $20.1393 \pm 0.0044$ & $0.04965 \pm 0.00001$ & $3.2 \pm 0.7$ & & $52,965.017 \pm 0.002$ \\
\hline & & & $20.6729 \pm 0.0044$ & $0.04837 \pm 0.00001$ & $2.3 \pm 0.7$ & & $52,965.024 \pm 0.002$ \\
\hline & & & $8.4227 \pm 0.0048$ & $0.11873 \pm 0.00007$ & $4.4 \pm 0.7$ & & $52,965.024 \pm 0.003$ \\
\hline & & & $7.9007 \pm 0.0045$ & $0.12657 \pm 0.00007$ & $3.3 \pm 0.7$ & & $52,965.079 \pm 0.004$ \\
\hline
\end{tabular}


TABLE 3

Radial Velocities of HD 8801

\begin{tabular}{|c|c|c|}
\hline Date (HJD $-2,400,000)$ & $\begin{array}{c}\text { Velocity } \\
\left(\mathrm{km} \mathrm{s}^{-1}\right)\end{array}$ & Reference \\
\hline $24,821.788 \ldots \ldots \ldots \ldots \ldots$ & -1.1 & 1 \\
\hline 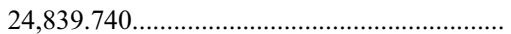 & 0.7 & 1 \\
\hline 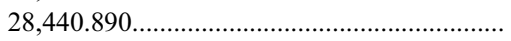 & 1.3 & 1 \\
\hline 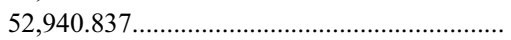 & -1.1 & 2 \\
\hline 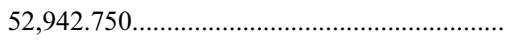 & 1.5 & 2 \\
\hline 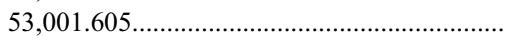 & 1.0 & 3 \\
\hline 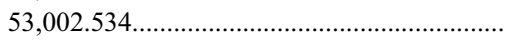 & 2.2 & 3 \\
\hline 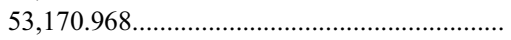 & 0.5 & 2 \\
\hline $53,171.970$ & 0.0 & 2 \\
\hline 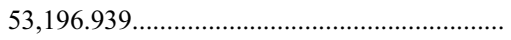 & 1.6 & 4 \\
\hline 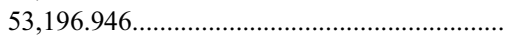 & 0.6 & 4 \\
\hline 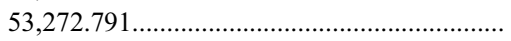 & 0.5 & 2 \\
\hline $53,275.779$ & 0.5 & 2 \\
\hline $53,277.891 \ldots \ldots \ldots \ldots$ & 1.3 & 2 \\
\hline
\end{tabular}

ReFERENCES.-(1) Harper 1937; (2) this paper; (3) D. Willmarth 2003, private communication; (4) J. Tomkin 2004, private communication.

produce a mean velocity of $0.8 \pm 0.3 \mathrm{~km} \mathrm{~s}^{-1}$. The small nightto-night velocity variations (Table 3 ) are consistent with pulsation effects (e.g., Fekel \& Henry 2003; Mathias et al. 2004). Harper (1937) reported three earlier velocities of HD 8801 (Table 3) that have an average of $0.3 \pm 1.2 \mathrm{~km} \mathrm{~s}^{-1}$, in good agreement with our new result. Thus, there is no indication from the 14 velocities that HD 8801 is a spectroscopic binary. While a very eccentric orbit with a period of several weeks or months cannot be completely ruled out, the observations to date indicate that HD 8801 is a single star.

\subsection{Am Star Classification of HD 8801}

Cowley et al. (1969) determined spectral classifications of about 1700 bright, northern A stars using spectrograms with a dispersion of $125 \AA \mathrm{mm}^{-1}$. In their survey they classified HD 8801 as an $A 7 \mathrm{~m}$ : marginal metallic-line star, where the spectral subclass refers to the K-line type. For their Am star classifications, they were guided by the qualitative definition of Roman et al. (1948) that a metallic-line star is one for which the K-line spectral type is "considerably" earlier than the type derived from its metal lines. More recently, Abt \& Morrell (1995) included HD 8801 in a spectroscopic survey of 1700 northern A-type stars. Abt \& Morrell (1995) noted that the higher dispersion of their spectrograms, $39 \AA \mathrm{mm}^{-1}$, enabled them to see faint lines better and resulted in the detection of a significantly greater number of peculiar stars than Cowley et al. (1969) had found. Abt \& Morrell (1995) classified HD 8801 as Am (A5/F1/F2), where the three spectral classes correspond to types for the $\mathrm{Ca} \mathrm{K}$, hydrogen, and metal lines, respectively. They designated it a classical Am star according to the definition of Abt \& Bidelman (1969), because the K-line and metal-line types differed by five or more subclasses. The classical Am stars and the early or hot Am stars lie on or near the main sequence and have $\mathrm{H}$-line types between $\mathrm{A} 3$ and $\mathrm{F} 1$ and between $\mathrm{A} 0$ and $\mathrm{A} 3$, respectively, while the evolved Am stars with luminosity classes IV and III are classified as $\delta$ Delphini or, perhaps preferably, $\rho$ Puppis stars (see discussion in Kurtz 2000).

We have both calcium and iron lines in our spectrograms of HD 8801 that were obtained at $6430 \AA$. Comparison with the spectra of several late-A and early-F dwarfs (see Fekel et al. 2003) indicates that the iron lines of HD 8801 are consistent with the spectrum of an F0 V star, while the calcium lines appear somewhat weaker than those in the same reference stars. Thus, the lines in this red wavelength region are consistent with Am star characteristics but do not distinguish it as a marginal or classical Am star. We determined $v \sin i=55 \pm 5 \mathrm{~km} \mathrm{~s}^{-1}$ with the procedure used by Fekel et al. (2003). Such a rotational velocity is typical of Am stars (Abt \& Morrell 1995).

Crawford et al. (1966) obtained Strömgren photometry and $\mathrm{H} \beta$ observations of HD 8801. A comparison of those results with the calibration of Crawford (1979) for A-type stars indicates that HD 8801 has a $\delta c_{1}=0.008$ and $\delta m_{1}=0.012$. The former value indicates that HD 8801 is firmly ensconced on the main sequence. Crawford (1979) notes that the A stars with the largest $m_{1}$ values are Am-type stars. The $\delta m_{1}$ value of $\mathrm{HD}$ 8801 is only a modest departure from the standard relation of Crawford (1979) and thus does not indicate particularly strong Am characteristics.

\section{DISCUSSION}

\subsection{Duplicity, Rotation, and Pulsation in Am Stars}

Early studies indicated that all metallic-line stars are members of binary systems (Roman et al. 1948; Abt 1961), are slow rotators (Roman et al. 1948; Slettebak 1954, 1955; Abt 1961; Abt \& Moyd 1973), and do not pulsate (Breger 1970, 1972; Kurtz et al. 1976). Abt $(1961,1965)$ surmised that the low rotational velocities of Am stars are primarily the result of the tendency toward synchronous rotation in binary systems but suggested other causes might be required to explain the slow rotation in the long-period Am binaries. Watson $(1970,1971)$ and Smith (1971) extended the ideas of Michaud (1970) and suggested that subsurface element diffusion processes in the stable outer layers permitted by slow rotation could produce the anomalous abundance patterns observed in Am stars. The calculations of Vauclair et al. (1974) demonstrated that diffusion results in the rapid settling of helium out of the $\mathrm{He}$ II ionization zone, which damps the driving mechanism for $\delta$ Sct pulsations and explains the absence of pulsations in the Am stars. Thus, the relationships between duplicity, rotation, pulsation, and metallicism seemed generally understood, at least qualitatively, by the mid-1970s.

More recent results, however, have revealed complications in this relatively simple picture. Abt \& Levy (1985) conducted an improved search for duplicity in a larger sample of Am stars and found that $25 \%$ of Am stars exhibited no evidence of binary companions out to periods of 1000 days. They suggested that tidal effects during pre-main-sequence contraction might provide the necessary braking for the intermediate-period binaries (10 days $<P<100$ days), while evolutionary expansion and its consequent rotational slowing (by up to a factor of 2) together with pre-main-sequence tidal effects could account for the slow rotation of Am stars in long-period binaries (100 days $<$ $P<1000$ days). Evolutionary expansion might even allow significant numbers of normal, single, A-type stars to become slowly rotating Am stars as they evolve off the main sequence. Recent studies of spectral peculiarities and rotation in A-type stars by Abt \& Morrell (1995) and Abt (2000) have confirmed the hypothesis that rotation alone determines whether a star will have normal or abnormal surface abundances, although Abt (1975) found no significant difference in rotational velocity or binary frequency between the classical Am and marginal Am: stars. Thus, some other factor or factors must determine whether a particular star exhibits classical or marginal abundance anomalies.

Kurtz (2000) reviewed the gradual recognition that Am star abundance anomalies can coexist with $\delta$ Sct pulsation in some 
cases. Kurtz (1976) first suggested that the anomalous abundance $\delta$ Del stars, with luminosity classes of IV and III, are evolved Am stars. Since some of the $\delta$ Del stars are also $\delta$ Sct pulsators, Kurtz (1976) concluded that either diffusion is a much stronger phenomenon than previously realized and can exist in a pulsating star, or diffusion is not the correct explanation for the abundance anomalies in Am stars. He preferred the first alternative because the diffusion hypothesis has been so successful in explaining Am star properties. Kurtz (1978) found lowamplitude $\delta$ Sct pulsations in two marginal metallic-line stars, HR 4594 and HR 8210, and concluded that, while classical Am stars do not pulsate, evolved Am stars and marginal Am: stars may pulsate. He also suggested that the presence of pulsation in the marginal Am: stars might be the missing factor from the Abt (1975) study that distinguishes the Am: stars from the Am stars. Kurtz (1984) added a third example of an Am: star with low-amplitude $\delta$ Sct pulsations, HR 3321, while Kurtz (1989) found the first example of pulsations in a classical Am star, HD 1097, which varies with a period of 81 minutes and a Johnson $B$ amplitude of 4.5 mmag. Kurtz (2000) listed three classical Am $-\delta$ Sct pulsators known by that time (HD 1097, HD 13038, and HD 13079), while Zhiping (2000) added a fourth (HD $27628=60$ Tau). Thus, the dichotomy between constant classical Am and pulsating marginal Am: stars proposed by Kurtz (1978) has been disrupted. The most extreme case of the coexistence of metallicism and pulsation is HD 40765 (Kurtz et al. 1995), a $\rho$ Pup ( $\delta$ Del) or evolved Am star with peak-to-peak light variations of $0.21 \mathrm{mag}$. Two additional evolved, pulsating Am stars (HD 98851 and HD 102480) have been discovered by Joshi et al. (2003). Therefore, it is clear that pulsation can occur in both main-sequence and evolved metallic-line stars.

Although Vauclair et al. (1974) showed that helium rapidly settles out of the He II ionization zone in stars exhibiting diffusion processes and thus shuts off the pulsation driving mechanism, Vauclair $(1976,1977)$ proposed a diffusion model that permits pulsation in stars with mild abundance anomalies. After a stable phase in which diffusion processes produce the Am star characteristics, turbulent motions increase and mix helium back into the He II ionization zone. Therefore, Am stars are predicted to evolve into $\delta$ Sct variables, and mild anomalies can be expected in evolved, low-amplitude pulsators. The theoretical pulsation models of Cox et al. (1979) demonstrated that metallicism and pulsation could exist simultaneously in low-helium $\delta$ Sct stars on the red side of the instability strip. Once most of the helium has settled out of the lower convection zone, and if it is not replaced by meridional circulation or turbulence, diffusion can act rapidly to establish the metallicism, while the residual helium in the ionization zone can drive the pulsations. Cox et al. (1979) predicted that low-helium $\delta$ Sct stars might even exist outside the red edge of the instability strip. Newer models of Am stars treating opacities and composition gradients in more detail have shown that a deeper mixing layer than previously realized must be present to account for the observed abundances (Turcotte 2002). These models predict pulsations in the more massive, as well as in the evolved, Am stars.

Thus, while diffusion remains the most generally accepted explanation for the properties of the metallic-line stars (Kurtz 2000), some difficulties remain. In particular, the presence of a pulsation-driving mechanism in some of the evolved Am stars, in some marginal Am: stars, and even in several classical Am stars must be understood in detail. Especially surprising is the case of HD 40765, in which extreme abundance anomalies implying an atmospheric stability with variations of fractions of a centimeter per second coexist with pulsational radial velocity
TABLE 4

Basic Properties of HD 8801

\begin{tabular}{|c|c|c|}
\hline Quantity & Value & Reference \\
\hline V & $6.42 \mathrm{mag}$ & 1 \\
\hline$B-V$ & $0.311 \mathrm{mag}$ & 1 \\
\hline Spectral type & Am (A5/F1/F2) & 2 \\
\hline 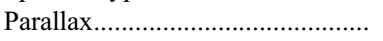 & $0.01791 \pm 0.00075$ & 1 \\
\hline Distance & $56 \pm 2 \mathrm{pc}$ & 1 \\
\hline$M_{v}$ & $2.68 \pm 0.09 \mathrm{mag}$ & 3 \\
\hline$M_{\mathrm{bol}}$ & $2.72 \pm 0.09 \mathrm{mag}$ & 3 \\
\hline$L$ & $6.5 \pm 0.6 L_{\odot}$ & 3 \\
\hline$T_{\text {eff }}$ & $7158 \pm 200 \mathrm{~K}$ & 3 \\
\hline$R$ & $1.7 \pm 0.1 R_{\odot}$ & 3 \\
\hline M & $1.55 \pm 0.1 M_{\odot}$ & 3 \\
\hline $\log g \ldots \ldots$ & $4.2 \mathrm{cgs}$ & 3 \\
\hline
\end{tabular}

References.-(1) Perryman et al. 1997; (2) Abt \& Morrell 1995; (3) this paper.

variations estimated to be around $14 \mathrm{~km} \mathrm{~s}^{-1}$. This problem has not been addressed theoretically (Kurtz 1998, 2000), and the seeming contradiction led Kurtz et al. (1995) to suggest that some other mechanism might be responsible for the strong metallicline spectrum in HD 40765 and, by inference, in other Am stars.

\subsection{The Status of HD 8801}

HD 8801 is the first example of a star pulsating with selfexcited $\gamma$ Dor and $\delta$ Sct frequencies. It is also interesting as a rare example of a pulsating, classical metallic-line star and is made even more unusual by the apparent lack of a binary companion. Thus, HD 8801 possesses a unique combination of physical properties. Only one other confirmed $\gamma$ Dor star, HD 221866 (Henry \& Fekel 2002), has been shown to have Am characteristics (Fekel et al. 2003), but Kaye et al. (2004) have found it to be a double-lined spectroscopic binary with an Am star primary and a normal, pulsating, early-F secondary. Consequently, HD 8801 is the first Am star known to have $\gamma$ Dor pulsations.

The radial velocity results provide no evidence that HD 8801 is a binary. However, our $v \sin i$ value of $55 \mathrm{~km} \mathrm{~s}^{-1}$ is somewhat smaller than the value of $78 \mathrm{~km} \mathrm{~s}^{-1}$ revised by Royer et al. (2002) from the original value of Abt \& Morrell (1995). This difference suggests that HD 8801 might be an unresolved double-lined binary. However, since HD 8801 lies near the zero-age main sequence (see below), its luminosity does not appear to be enhanced by the presence of a companion of similar brightness. Based on its lack of velocity variations and its position in the H-R diagram, we assume that HD 8801 is a single star.

We have computed the basic properties of HD 8801 with the $V$ magnitude, $B-V$ color index, and parallax from the Hipparcos catalog (Perryman et al. 1997), Table 3 of Flower (1996), and the Stefan-Boltzmann law. The results are listed in Table 4. We have compared the computed luminosity and effective temperature with the solar composition evolutionary tracks of Schaller et al. (1992). HD 8801 is positioned quite close to the zero-age main sequence and has an estimated mass of $1.55 \pm 0.1 M_{\odot}$, resulting in $\log g=4.2$. Our effective temperature and $\log g$ values are in reasonable agreement with those determined by Guthrie (1987), who used uvby and $\mathrm{H} \beta$ photometry and the grids of Moon \& Dworetsky (1985) to determine those quantities.

The $B-V$ and $M_{v}$ values for HD 8801 from Table 4 are plotted in the H-R diagram of Figure 5, adapted from Figure 28 of Henry \& Fekel (2003). The figure shows the location of all 42 confirmed $\gamma$ Dor variables listed in Table 6 of Henry \& Fekel (2003), along with the observed $\delta$ Sct and $\gamma$ Dor instability 


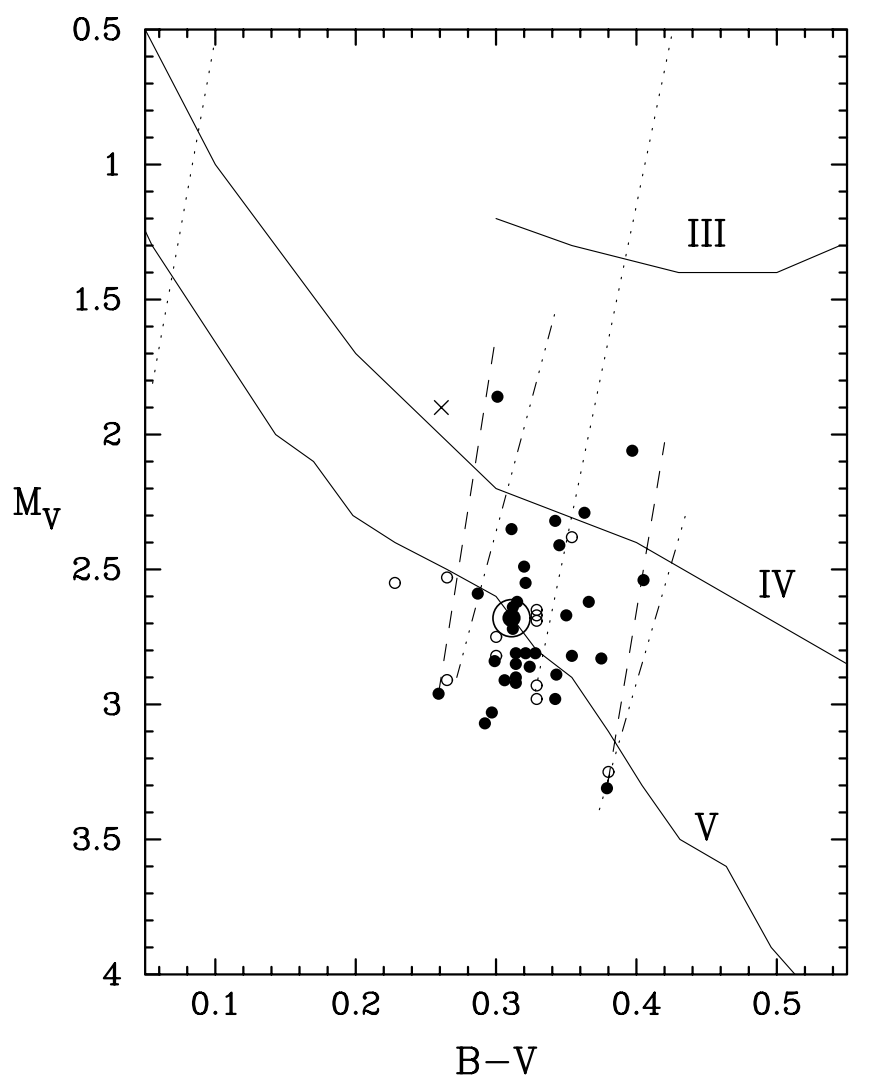

FIG. 5.-Position of HD 8801 (large circled point) in the H-R diagram, adapted from Fig. 28 of Henry \& Fekel (2003). All 42 confirmed $\gamma$ Dor variables from Henry \& Fekel (2003) are shown, along with the observed $\delta$ Sct and $\gamma$ Dor instability strips (dotted and dashed lines, respectively) and the theoretical instability strip for $\gamma$ Dor stars computed by Warner et al. (2003; triple-dotdashed lines). The $\gamma$ Dor stars with well-determined locations in the diagram are plotted as filled circles, while those with somewhat greater uncertainty (mostly components of double-lined spectroscopic binary systems) are plotted as open circles. HD 209295 is plotted as a cross, since its $\gamma$ Dor pulsation is tidally excited. HD 8801 lies on the main sequence and within both the $\delta$ Sct and $\gamma$ Dor instability strips.

strips (dotted and dashed lines, respectively) and the theoretical instability strip for $\gamma$ Dor stars computed by Warner et al. (2003; triple-dot-dashed lines). HD 8801 lies on the main sequence within both the $\delta$ Sct and $\gamma$ Dor instability strips.

HD 8801 also appears in Figure 2 of Kurtz (1978), which plots the $\left(\beta, M_{v}\right)$ plane for the sample of $44 \mathrm{Am}$ : stars from Cowley et al. (1969). HD 8801 lies among a cluster of four stars in the lower right of that figure, near the zero-age main sequence and the red edge of the $\delta$ Sct instability strip. Two of those four stars are HR 4594 and HR 8210, which Kurtz (1978) found to be low-amplitude $\delta$ Sct stars. It is interesting that we have also found the fourth star, HR 4543, to be a low-amplitude variable in our volume-limited survey of $114 \gamma$ Dor candidates (G. W. Henry et al. 2005, in preparation), although we have not yet determined its period. The pulsating classical Am stars HD 1097, HD 13038, HD 13079, and HD 27628 mentioned above also lie in the lower right corner of the $\delta$ Sct instability strip near these four stars. Therefore, it appears that the rare, pulsating, classical Am and Am: stars are concentrated in this part of the H-R diagram. As
TABLE 5

$Q$-VALUES FOR HD 8801

\begin{tabular}{|c|c|c|c|}
\hline $\begin{array}{l}\text { Period } \\
\text { (days) }\end{array}$ & $\log Q$ & $\begin{array}{c}Q \\
\text { (days) }\end{array}$ & Variability Class \\
\hline 0.0484 & -1.547 & 0.028 & $\delta$ Sct \\
\hline $0.0496 \ldots \ldots \ldots$ & -1.537 & 0.029 & $\delta$ Sct \\
\hline $0.1188 \ldots \ldots$ & -1.157 & 0.070 & $\delta$ Sct \\
\hline $0.1265 \ldots \ldots$ & -1.130 & 0.074 & $\delta$ Sct \\
\hline $0.3523 \ldots \ldots \ldots \ldots \ldots$ & -0.685 & 0.206 & $\gamma$ Dor \\
\hline $0.4033 \ldots \ldots$ & -0.626 & 0.236 & $\gamma$ Dor \\
\hline
\end{tabular}

noted previously, Cox et al. (1979) concluded that metallicism and pulsation could coexist in low-helium stars near the red edge of the instability strip, but the models of Turcotte (2002) predicted pulsations only in more massive and more evolved Am stars.

The projected rotational velocity of $55 \mathrm{~km} \mathrm{~s}^{-1}$ for HD 8801 is typical for Am stars, which, as a group, rotate more slowly than normal A stars. The position of HD 8801 on the main sequence argues against evolutionary expansion and rotational spin-down as the explanation for its low rotational velocity compared to normal A stars, as suggested by Abt \& Levy (1985) as the reason for Am spectral characteristics in long-period binaries and possibly in some single stars. Now that Hipparcos parallaxes are available for the 11 constant-velocity stars in the sample of 55 Am stars in Abt \& Levy (1985), we computed their $M_{v}$ values and found five to be main-sequence stars, five to be subgiants, and one to be a giant. Thus, evolutionary effects do not seem able to account for all cases of slow rotation in single Am stars. Perhaps some additional factor, such as the presence of planetary systems, is responsible for the slow rotation in some single metallic-line stars; however, this is purely speculative at this point, since such early-type stars have not been included in existing searches for extrasolar planets (see, e.g., Wright et al. 2004).

Handler \& Shobbrook (2002) demonstrated that, although there is a near overlap of the longest $\delta$ Sct periods and the shortest $\gamma$ Dor periods, the ranges of pulsation constants of the two variable types are clearly separated. With the equation given by Handler \& Shobbrook (2002) and the basic parameters listed in our Table 4, we have computed the pulsation constant $Q$ for each of the six periods in HD 8801 (Table 5). The $\log Q$ values for the two longest periods fall within the range for the $\gamma$ Dor stars, while the values for the two shortest periods fall within the range for the $\delta$ Sct variables. The $\log Q$ values for the two intermediate periods of HD 8801 are just slightly outside the range of values for the $\delta$ Sct stars.

We greatly appreciate the help of $\mathrm{H}$. Abt and D. Willmarth as well as G. Handler and J. Tomkin, who acquired and measured four of the spectra discussed in this paper. We also thank L. Boyd for his continuing support as Director of Fairborn Observatory and G. Handler for comments on a draft of this paper. This research is supported by NASA grant NCC5-511 and NSF grant HRD-9706268. 
Breger, M. 1970, ApJ, 162, 597 1972, ApJ, 176, 373

2000, in ASP Conf. Ser. 210, Delta Scuti and Related Stars, ed. M. Breger \& M. H. Montgomery (San Francisco: ASP), 3

Breger, M., \& Beichbuchner, F. 1996, A\&A, 313, 851

Chevalier, C. 1971, A\&A, 14, 24

Cowley, A., Cowley, C., Jaschek, M., \& Jaschek, C. 1969, AJ, 74, 375

Cox, A. N., King, D. S., \& Hodson, S. W. 1979, ApJ, 231, 798

Crawford, D. L. 1979, AJ, 84, 1858

Crawford, D. L., Barnes, J. V., Faure, B. Q., Golson, J. C., \& Perry, C. L. 1966, AJ, 71, 709

Eaton, J. A., Henry, G. W., \& Fekel, F. C. 2003, in The Future of Small Telescopes in the New Millennium, Vol. II: The Telescopes We Use, ed. T. D. Oswalt (Dordrecht: Kluwer), 189

Fekel, F. C., \& Henry, G. W. 2003, AJ, 125, 2156

Fekel, F. C., Warner, P. B., \& Kaye, A. B. 2003, AJ, 125, 2196

Fitzpatrick, M. J. 1993, in ASP Conf. Ser. 52, Astronomical Data Analysis Software and Systems II, ed. R. J. Hanisch, R. V. J. Brissenden, \& J. Barnes (San Francisco: ASP), 472

Flower, P. J. 1996, ApJ, 469, 355

Guthrie, B. N. G. 1987, MNRAS, 226, 361

Guzik, J. A., Kaye, A. B., Bradley, P. A., Cox, A. N., \& Neuforge, C. 2000, ApJ, 542, L57

Handler, G. 1999, MNRAS, 309, L19

Handler, G., \& Shobbrook, R. R. 2002, MNRAS, 333, 251

Handler, G., et al. 2002, MNRAS, 333, 262

Harper, W. E. 1937, Publ. Dom. Astrophys. Obs., 7, 1

Henry, G. W. 1999, PASP, 111, 845

Henry, G. W., \& Fekel, F. C. 2002, PASP, 114, 988

2003, AJ, 126, 3058

Henry, G. W., Fekel, F. C., \& Henry, S. M. 2004, AJ, 127, 1720

Henry, G. W., Fekel, F. C., Kaye, A. B., \& Kaul, A. 2001, AJ, 122, 3383

Joshi, S., et al. 2003, MNRAS, 344, 431

Kaye, A. B., Gray, R. O., \& Griffin, R. F. 2004, PASP, 116, 558

Kaye, A. B., Handler, G., Krisciunas, K., Poretti, E., \& Zerbi, F. M. 1999, PASP, 111,840

Kurtz, D. W. 1976, ApJS, 32, 651

1978, ApJ, 221, 869

1984, MNRAS, 206, 253

1989, MNRAS, 238, 1077
Kurtz, D. W. 1998, in ASP Conf. Ser. 135, A Half-Century of Stellar Pulsation Interpretations, ed. P. A. Bradley \& J. A. Guzik (San Francisco: ASP), 420 . 2000, in ASP Conf. Ser. 210, Delta Scuti and Related Stars, ed. M. Breger \& M. H. Montgomery (San Francisco: ASP), 287

- 2002, in ASP Conf. Ser. 259, Radial and Nonradial Pulsations as Probes of Stellar Physics, ed. C. Aerts, T. R. Bedding, \& J. ChristensenDalsgaard (San Francisco: ASP), 639 2003, Ap\&SS, 284, 29

Kurtz, D. W., Breger, M., Evans, S. W., \& Sandmann, W. H. 1976, ApJ, 207, 181 Kurtz, D. W., Garrison, R. F., Koen, C., Hofmann, G. F., \& Viranna, N. B. 1995, MNRAS, 276, 199

Loumos, G. L., \& Deeming, T. J. 1978, Ap\&SS, 56, 285

Mathias, P., et al. 2004, A\&A, 417, 189

Michaud, G. 1970, ApJ, 160, 641

Moon, T. T., \& Dworetsky, M. M. 1985, MNRAS, 217, 305

Perryman, M. A. C., et al. 1997, The Hipparcos and Tycho Catalogues (ESA SP-1200; Noordwijk: ESA)

Rodríguez, E., López-González, M. J., \& López de Coca, P. 2000, A\&AS, 144, 469

Roman, N. G., Morgan, W. W., \& Eggen, O. J. 1948, ApJ, 107, 107

Royer, F., Grenier, S., Baylac, M.-O., Gómez, A. E., \& Zorec, J. 2002, A\&A, 393, 897

Scarfe, C. D., Batten, A. H., \& Fletcher, J. M. 1990, Publ. Dom. Astrophys. Obs., 18, 21

Schaller, G., Schaerer, D., Meynet, G., \& Maeder, A. 1992, A\&AS, 96, 269

Slettebak, A. 1954, ApJ, 119, 146 1955, ApJ, 121, 653

Smith, M. A. 1971, A\&A, 11, 325

Turcotte, S. 2002, in ASP Conf. Ser. 259, Radial and Nonradial Pulsations as Probes of Stellar Physics, ed. C. Aerts, T. R. Bedding, \& J. ChristensenDalsgaard (San Francisco: ASP), 258

Vaniĉek, P. 1971, Ap\&SS, 12, 10

Vauclair, G. 1976, A\&A, 50, 435 1977, A\&A, 55, 147

Vauclair, G., Vauclair, S., \& Pamjatnikh, A. 1974, A\&A, 31, 63

Warner, P. B., Kaye, A. B., \& Guzik, J. A. 2003, ApJ, 593, 1049

Watson, W. D. 1970, ApJ, 162, L45 1971, A\&A, 13, 263

Wright, J. T., Marcy, G. W., Butler, R. P., \& Vogt, S. S. 2004, ApJS, 152, 261

Zhiping, L. 2000, A\&A, 360, 185

Note added in proof.-HD 8801 was placed on the observing menu of the new Tennessee State University $2 \mathrm{~m}$ automatic spectroscopic telescope at Fairborn Observatory in Arizona. Between 2004 January and 2005 February, 17 echelle spectra covering the wavelength range 4900-7200 $\AA$ were obtained. Velocities, measured relative to one spectrum of HD 8801 , were provided to us by $\mathrm{M}$. Williamson (2005, private communication). The 16 different velocities span a total range of $3.1 \mathrm{~km} \mathrm{~s}$ and have an average of $0.15 \pm 0.18$. This result is consistent with our conclusion that HD 8801 is a single star with modest velocity changes caused by pulsation. 